\title{
Remissão total de linfoma multicêntrico em cão com o protocolo Madison-Wisconsin:
}

\section{Relato de caso}

\author{
Total remission of multicentric lymphoma in dog with the Madison-Wisconsin protocol: Case \\ report
}

Remisión total del linfoma multicêntrico em perro com el protocolo Madion-Wisconsin: Reporte de

\author{
Glenda Roberta Freire Lima \\ ORCID: https://orcid.org/0000-0002-3867-7389 \\ Universidade Estadual do Ceará, Brasil \\ E-mail: glendaroberta.medvet@gmail.com \\ Ana Thays dos Santos da Silva \\ ORCID: https://orcid.org/0000-0001-7096-0080 \\ Universidade Estadual do Ceará, Brasil \\ E-mail: anathays1234@gmail.com \\ Vitória Maria Jorge de Araújo \\ ORCID: https://orcid.org/0000-0001-6489-6959 \\ Universidade Estadual do Ceará, Brasil \\ E-mail: vitoria.m.arauj@ gmail.com \\ Germano Gonçalves Teixeira \\ ORCID: https://orcid.org/0000-0002-6586-1819 \\ Universidade Estadual do Ceará, Brasil \\ E-mail: germanoggt06@gmail.com \\ Ana Beatriz dos Santos Mendes \\ ORCID: https://orcid.org/0000-0002-3647-4465 \\ Universidade Estadual do Ceará, Brasil \\ E-mail: anabiamedvet@gmail.com \\ Francisco Douglas Lima Anastácio \\ ORCID: https://orcid.org/0000-0001-6943-4796 \\ Universidade Estadual do Ceará, Brasil \\ E-mail: douglasanastacio7@gmail.com \\ Renata Borges da Silva \\ ORCID: https://orcid.org/0000-0001-8246-747X \\ Universidade Estadual do Ceará, Brasil \\ E-mail: renataelborges@gmail.com \\ Breno Queiroz Pinheiro \\ ORCID: https://orcid.org/0000-0001-6363-6952 \\ Universidade Estadual do Ceará, Brasil \\ E-mail: breno.queiroz@uece.br
}

\begin{abstract}
Resumo
O linfoma é um distúrbio do sistema hematopoiético cuja etiologia envolve a proliferação desordenada de células linfóides, no qual o linfoma multicêntrico é caracterizado pelo aumento generalizado dos linfonodos. O protocolo Madison-Wisconsin é uma das poliquimioterapias mais utilizadas no tratamento de linfomas multicêntrico em cães, sendo constituída pelos fármacos ciclofosfamida, doxorrubicina, vincristina e prednisona, e administrados de forma alternada ao longo de 19 semanas. O presente trabalho teve como objetivo relatar a eficiência do protocolo terapêutico Madison-Wisconsin na remissão total de um linfoma multicêntrico em um cão. $O$ animal atendido foi um cão da raça Bulldog Francês, que apresentava aumento dos linfonodos submandibulares, pré-escapulares, axilares, inguinais e poplíteos e, ao exame físico, o aumento em região mesogástrica sugeriu esplenomegalia. O exame citológico dos linfonodos submandibular e poplíteo foi compatível com linfoma. No exame ultrassonográfico detectou-se aumento acentuado do baço e fígado, caracterizando o quadro de hepatoesplenomegalia. No ecodopplercardiograma foi possível observar um aumento no átrio esquerdo. Houve acompanhamento hematológico do paciente durante tratamento quimioterápico e ao longo do curso terapêutico o animal apresentou alterações no hemograma, como trombocitose e neutropenia, o que requereu um tratamento adicional. No exame bioquímico, houve aumento da ALT. Após o término da quimioterapia o animal apresentou remissão total da neoplasia demonstrando o sucesso terapêutico do protocolo de Madison-Wisconsin sobre o linfoma.
\end{abstract}

Palavras-chave: Linfoma; Quimioterapia; Tratamento. 


\begin{abstract}
Lymphoma is a disorder of the hematopoietic system whose etiology involves a disordered proliferation of lymphoid cells, in which multicentric lymphoma is triggered by generalized lymph node enlargement. The Madison-Wisconsin protocol is one of the most used polychemotherapies in the treatment of multicenter lymphomas in dogs, being verified by the drugs cyclophosphamide, doxorubicin, vincristine and prednisone, and administered alternately over 19 weeks. This study aimed to report the efficiency of the Madison-Wisconsin therapeutic protocol in the total remission of a multicentric lymphoma in a dog. The animal assisted was a French Bulldog breed dog, which presented enlargement of the submandibular, pre-scapular, axillary, inguinal and popliteal lymph nodes and, on physical examination, the enlargement in the mesogastric region suggested splenomegaly. Cytological examination of the submandibular and popliteal lymph nodes was compatible with lymphoma. Ultrasonographic examination detected a marked increase in the spleen and liver, characterizing hepatosplenomegaly. No Doppler echocardiogram was possible to observe an increase in the left atrium. There was hematological follow-up of the patient during chemotherapy treatment and throughout the therapeutic course or changes in animals in the blood count, such as thrombocytosis and neutropenia, which required additional treatment. Without biochemical examination, there was an increase in ALT. After the end of chemotherapy, the animal presented complete remission of the neoplasm, demonstrating the therapeutic success of the Madison-Wisconsin protocol on lymphoma.
\end{abstract}

Keywords: Lymphoma; Chemotherapy; Treatment.

\title{
Resumen
}

El linfoma es un trastorno del sistema hematopoyético cuya etiología implica una proliferación desordenada de células linfoides, en el que el linfoma multicéntrico se desencadena por agrandamiento generalizado de los ganglios linfáticos. El protocolo Madison-Wisconsin es una de las poliquimioterapias más utilizadas en el tratamiento de linfomas multicéntricos en perros, siendo verificado por los fármacos ciclofosfamida, doxorrubicina, vincristina y prednisona, y administrado alternativamente durante 19 semanas. Este estudio tuvo como objetivo informar de la eficacia del protocolo terapéutico de Madison-Wisconsin en la remisión total de un linfoma multicéntrico en un perro. El animal asistido fue un perro de raza Bulldog Francés, el cual presentó agrandamiento de los ganglios linfáticos submandibulares, preescapulares, axilares, inguinales y poplíteos y, al examen físico, el agrandamiento en la región mesogástrica sugirió esplenomegalia. El examen citológico de los ganglios linfáticos submandibulares y poplíteos fue compatible con linfoma. El examen ecográfico detectó un marcado aumento en el bazo y el hígado, caracterizando la hepatoesplenomegalia. Ningún ecocardiograma Doppler fue posible para observar un aumento en la aurícula izquierda. Se realizó seguimiento hematológico del paciente durante el tratamiento quimioterápico y a lo largo del curso terapéutico o cambios en el hemograma en animales, como trombocitosis y neutropenia, que requirieron tratamiento adicional. Sin examen bioquímico, hubo un aumento de ALT. Tras finalizar la quimioterapia, el animal presentó remisión completa de la neoplasia, demostrando el éxito terapéutico del protocolo Madison-Wisconsin sobre linfoma.

Palabras clave: Linfoma; Quimioterapia; Tratamiento.

\section{Introdução}

O linfoma é caracterizado por uma neoplasia maligna do sistema hematopoiético cuja etiologia envolve uma proliferação desordenada de células linfóides de etiologia multifatorial, que possui elevada incidência em cães (Vail, Thamm, \& Liptack, 2020). Esta patologia acomete cães de meia idade a idosos, sendo as raças Boxer, Rottweiler, Poodle, Chow Chow, Beagle, Basset Hound, Pastor Alemão, São Bernardo, Scottish Terrier, Airedale Terrier e Bulldog as que apresentam maior ocorrência (Daleck \& De Nardi, 2016).

De acordo com sua localização clínico-anatômica é classificado como mediastinal, gastrointestinal, extranodal, cutânea e multicêntrica, onde esta última representa cerca de $80 \%$ dos casos em cães (Vail et al., 2020). O linfoma multicêntrico é caracterizado pelo aumento generalizado dos linfonodos, inicialmente submandibulares, pré-escapulares e axilares, progredindo para linfadenomegalia generalizada, acompanhados de desconforto, apatia, febre, hiporexia e anorexia (Valli, Bienzle, \& Meuten, 2017). O animal também pode apresentar edema localizado, caso ocorra compressão de algum vaso linfático (Daleck \& De Nardi, 2016). O linfoma multicêntrico tem como manifestação secundária mais comum infiltração hepatoesplênica e em casos mais avançados pode ocorrer envolvimento pulmonar com infiltração difusa (Vail et al., 2020). Nos exames hematológicos de animais com linfoma multicêntrico são observadas alterações variadas, mas a anemia é uma das alterações mais comumente observadas (Capua et al., 2011). 
A quimioterapia é uma das modalidades terapêuticas mais utilizadas no tratamento dessa patologia, visto que essa doença se dá de forma disseminada e sistêmica (Beraldo, Varzim, \& Pulz, 2020). Dentre as opções, o protocolo de MadisonWisconsin, também conhecido como UW-19, é um protocolo desenvolvido pela Universidade de Wisconsin Madison, e é reconhecido como uma das poliquimioterapias mais utilizadas no tratamento de linfomas multicêntrico em cães, sendo constituído por um tratamento de 19 semanas a base de ciclofosfamida, doxorrubicina, vincristina e prednisona (Hosoya et al., 2007; Marconato, 2011).

Os fármacos utilizados no protocolo UW-19 são administrados de forma alternada ao longo das 19 semanas, enquanto a prednisona se faz de forma contínua e reduzida ao longo das primeiras 4 semanas de tratamento (Burton, Mayer, \& Thamm, 2012). A taxa de remissão completa do linfoma multicêntrico, através do retratamento pelo protocolo Madison-Wisconsin pode atingir uma média de aproximadamente 78-90\% dos casos (Flory et al., 2011). Com isso, o objetivo deste trabalho é relatar a eficiência do protocolo terapêutico de Madison-Wisconsin para a remissão total de um caso de linfoma multicêntrico canino.

\section{Metodologia}

Esse artigo trata-se de um relato de caso, com uma abordagem descritiva e qualitativa, de acordo com Pereira et al. (2018). Todas as informações foram colhidas através do prontuário online do paciente, com a disponibilização de exames de imagem para discussão. No que se relacionam aos aspectos éticos, o uso de informações sigilosas do animal, assim como de seu tutor foram omitidas.

\section{Relato de Caso}

Paciente da raça Bulldog Francês, não castrado, sete anos de idade, apresentando perda de peso e apatia. Ao exame físico os linfonodos submandibulares, pré-escapulares, axilares, inguinais e poplíteos estavam aumentados, e os demais parâmetros estavam dentro da normalidade. À palpação abdominal, o aumento em região mesogástrica sugeriu esplenomegalia. Como exames complementares realizaram-se teste rápido SNAP 4DX Plus ${ }^{\circledR}$, útil para diagnóstico de dirofilariose, erliquiose, anaplasmose e doença de Lyme cujo resultado foi negativo. Foram solicitados também exames de imagem como, ultrassonografia abdominal total e radiografia torácica em três projeções, bem como hemograma completo, avaliação bioquímica renal e hepática para análise da ALT, ureia, creatinina, fosfatase alcalina, cálcio, fósforo e glicose, além da citologia de linfonodos submandibular e poplíteo, que foi sugestiva de linfoma.

O tratamento terapêutico instituído foi baseado no protocolo quimioterápico Madison-Wisconsin, que está esquematizado na Tabela 1. A cada semana o paciente era acompanhado através de exames hematológicos e bioquímicos prévios à administração dos quimioterápicos. 
Tabela 1: Protocolo Madison-Wisconsin para cães.

\begin{tabular}{|c|c|c|c|c|}
\hline $\begin{array}{c}\text { Semana de } \\
\text { administração }\end{array}$ & $\begin{array}{c}\text { Prednisona } \\
\text { VO } *\end{array}$ & $\begin{array}{l}\text { Vincristina } \\
0,7 \mathrm{mg} / \mathrm{m}^{2}, \text { IV }\end{array}$ & $\begin{array}{c}\text { Ciclofosfamida } 250 \\
\mathrm{mg} / \mathrm{m}^{2}, \mathrm{VO}\end{array}$ & $\begin{array}{c}\begin{array}{c}\text { Doxorrubicina } 30 \\
\mathrm{mg} / \mathrm{m}^{2}, \text { IV }\end{array} \\
\end{array}$ \\
\hline $\mathbf{1}^{\mathrm{a}}$ & $\mathrm{X}$ & $\mathrm{X}$ & & \\
\hline $2^{a}$ & $\mathrm{X}$ & & $\mathrm{X}$ & \\
\hline $3^{\mathbf{a}}$ & $\mathrm{X}$ & $\mathrm{X}$ & & \\
\hline $4^{a}$ & $\mathrm{X}$ & & & $X$ \\
\hline $6^{a}$ & & $X$ & & \\
\hline $7^{a}$ & & & $X$ & \\
\hline $8^{a}$ & & $X$ & & \\
\hline $9^{a}$ & & & & $X$ \\
\hline $11^{\mathrm{a}}$ & & $X$ & & \\
\hline $12^{\mathrm{a}}$ & & & $\mathrm{X}$ & \\
\hline $13^{\mathrm{a}}$ & & $\mathrm{X}$ & & \\
\hline $14^{\mathrm{a}}$ & & & & $\mathrm{X}$ \\
\hline $16^{\mathrm{a}}$ & & $X$ & & \\
\hline $17^{\mathrm{a}}$ & & & $X$ & \\
\hline $18^{\mathrm{a}}$ & & $X$ & & \\
\hline $19^{a}$ & & & & $X$ \\
\hline
\end{tabular}

*Doses decrescentes de $2 \mathrm{mg} / \mathrm{Kg}, 1,5 \mathrm{mg} / \mathrm{Kg}, 1 \mathrm{mg} / \mathrm{Kg}$ e $0,5 \mathrm{mg} / \mathrm{Kg}$ por semana de administração. Adaptado: Daleck \& De Nardi (2016).

Não houve alteração nos exames, até a sexta semana do tratamento, em que o hemograma do animal revelou uma neutropenia (neutrófilos segmentados $1600 / \mu \mathrm{L}$ ), sendo prescrito um imunoestimulante (Promun Dog®) onde se observou melhora do quadro. Na décima primeira semana do tratamento, os linfonodos submandibulares estavam aumentados e foi realizada citologia aspirativa (linfonodo submandibular direito) para melhor avaliação do quadro.

\section{Resultados e Discussão}

Os resultados da punção não aspirativa por agulha fina (PNAAF) realizada em linfonodo submandibular direito e linfonodo poplíteo esquerdo, no momento da primeira avaliação clínica inicial, revelaram amostra de elevada celularidade monomórfica composta de células redondas, por vezes pleomórficas, apresentando citoplasma intensamente basofílico delimitado com elevada relação nuclear. Núcleo de formato redondo a oval, pleomórfico, cromatina frouxa, com presença de macronucléolos, por vezes múltiplos, indicando se tratar de linfoma multicêntrico. A citologia é um procedimento minimamente invasivo que oferece vantagens, como diagnóstico rápido com baixo índice de falso negativo e de fácil realização (Daleck \& De Nardi, 2016).

Vail et al. (2020) citam que outras causas de linfadenopatia incluem infecções causadas por protozoários (Toxoplasma $s p .$, Leishmania sp.) e rickettsias (Ehrlichia sp). No presente caso, o diagnóstico diferencial foi realizado por meio do resultado negativo do exame Snap 4DX para Anaplasma, Erliquia, Lyme e Dirofilária, permitindo descartar esses possíveis agentes causais. Ademais, a análise eletrolítica indicou que o sódio, potássio e cloreto estavam em valores dentro dos limites de referência.

Pelo exame ultrassonográfico, foi possível observar o baço e o fígado (Figura 1) com suas dimensões aumentadas, indicando hepatoesplenomegalia, corroborando com (Daleck \& De Nardi, 2016), que citam a visualização de hepatoesplenomegalia, um achado comum em linfomas na sua forma multicêntrica em cães, sendo a escolha desse exame de fundamental importância, pois serve auxílio na detecção de envolvimento hepático, esplênico, intestinal e dos linfonodos mesentéricos. Em estudo de Eberhardt et al. (2015) a esplenomegalia foi um achado comum em cães acometidos de linfoma multicêntrico canino, associando tal alteração a maior duração da doença e o posterior comprometimento deste órgão. Ademais, no exame ultrassonográfico foi possível observar nódulos hipoecóicos no baço semelhantes aos casos de linfoma 
abordados por Oliveira (2019) e por Laoharatchatathanin, Klomsatid e Thermtong (2014). Apesar dos distúrbios esplênicos serem comuns em cães de meia-idade e idosos, onde há esplenomegalia causada pela nodulação independentemente da gravidade da doença (Archer \& Sullivant, 2018), é sabido que muitos dos cães diagnosticados com linfoma multicêntrico já se encontram com o estádio elevado da doença e consequentemente comprometimento esplênico (Jia, Hou, Meng, \& Ma, 2016). Desse modo, uma alternativa seria a realização de biópsias ou punção aspirativa por agulha fina (PAAF) (Zamokas et al., 2016) para avaliação histopatológica e imuno-histoquímica, como foi efetuado por Jia et al. (2016) e Mazaro et al. (2018), para detectar ou excluir a presença de nodulação de linfoma maligno neste órgão, entretanto os tutores do paciente não concordaram com a realização desse procedimento.

Figura 1: Ultrassom abdominal evidenciando hepatoesplenomegalia e focos hipoecóicos no baço. Aumento de tamanho do baço em A. Aumento do tamanho do fígado em B.

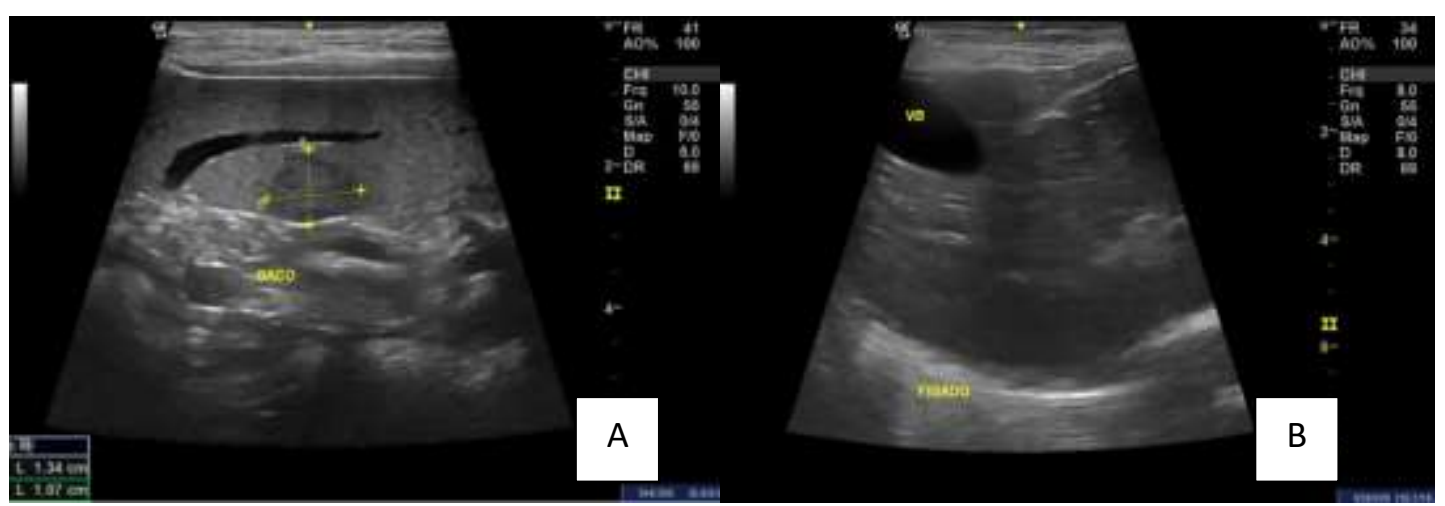

Fonte: Focus Diagnóstico Fortaleza (2019).

Dentre outros exames de imagem, o ecodopplercardiograma detectou-se aumento atrial esquerdo, sem repercussões hemodinâmicas. O ecocardiograma é uma importante forma para o diagnóstico de linfoma cardíaco, mas também deve ser realizado antes da administração de quimioterápicos cardiotóxicos, como a doxorrubicina, principalmente em animais com histórico de doença cardíaca ou raças que apresentam pré-disposição para cardiomiopatia (Daleck \& De Nardi, 2016). Apesar do aumento atrial evidenciado no exame, a administração da doxorrubicina não foi contra indicada, visto que essa alteração não repercutiu hemodinamicamente no animal.

O linfoma multicêntrico pode ser classificado em 5 estágios de acordo com a Organização Mundial da Saúde (OMS), sendo classificado como estágio 1 , quando ocorre o acometimento de um único linfonodo ou tecido linfóide; estágio 2, quando ocorre o envolvimento de outros linfonodos em uma região do corpo, como os regionais; o estágio 3 ocorre quando se observa o aumento generalizado dos linfonodos sem resposta dolorosa; estágio 4 quando se tem o envolvimento do fígado e/ou baço; e o último estágio com o acometimento da circulação sanguínea, com ou sem envolvimento da medula óssea (Zandvliet, 2016). $\mathrm{O}$ estadiamento do linfoma e o conhecimento a respeito do linfócito acometido, são importantes ferramentas para definir o prognóstico do paciente e escolha do tratamento a ser utilizado (Biller et al., 2016). Nesse caso, devido às alterações em baço e fígado, provavelmente o animal se encontrava em estádio 4. Porém, não foi realizado exame citológico para a confirmação de possíveis infiltrados da neoplasia nestes órgãos.

A poliquimioterapia (PQT) realizada pelo protocolo Madison-Wisconsin, é uma tentativa de aplicar tratamento antitumoral a todas as células resistentes, ou seja, a utilização de mais de um agente citostático em combinação, sendo eficaz no tratamento de cães com linfoma. Em geral, a remissão do linfoma ocorre em torno de $80 \%$ e varia entre quatro a oito meses (Daleck \& De Nardi, 2016). Após o dia 07 de dezembro de 2019, com o término do protocolo de tratamento quimioterápico de Madison-Wisconsin, que teve início no dia 08 de agosto do mesmo ano, foram realizados novos exames para o reestadiamento 
da neoplasia: hemograma completo, avaliação bioquímica sérica renal e hepática, ecodopplercardiograma, radiografia torácica e ultrassonografia abdominal. Estes exames foram repetidos após um mês, com exceção do ecodopplercardiograma. Novamente, após três meses, os mesmos exames foram repetidos. O acompanhamento do animal se deu até julho de 2020 e nenhum dos exames apresentou alterações dignas de registro. O animal apresentou-se estável, com remissão completa do linfoma multicêntrico, demonstrando um bom estado de saúde. Essa modalidade quimioterápica cursa com bons resultados, refletidos no maior tempo de sobrevida dos animais submetidos e melhor taxa de resposta ao tratamento (Garret, Thamm, Chun, Dudley, \& Vail, 2002).

No hemograma, ao longo do tratamento, foram evidenciados anemia, leucopenia por neutropenia, e discreta trombocitose, corroborando com Cápua et al. (2011), que descreveram estas alterações hematológicas em cães acometidos pelo linfoma multicêntrico e tratados com o protocolo Madison-Wincosin. Neel, Synder e Grindem (2012) apontam a trombocitose como uma ocorrência secundária à administração da vincristina e de glicocorticóides em cães. Na avaliação bioquímica constatou-se o aumento da enzima alanina aminotransferase (ALT), provavelmente em decorrência da hepatotoxicidade provocada pela prednisona, vincristina, doxorrubicina e ciclofosfamida, drogas que apresentam metabolização essencialmente hepática (Pereira et al., 2011; Silveira, 2016; Nelson \& Couto, 2015).

$\mathrm{O}$ tratamento quimioterápico do presente caso consistiu no protocolo de Wisconsin-Madison que é uma adaptação do protocolo CHOP (Chun, 2019), uma combinação de vincristina, prednisolona, ciclofosfamida e doxorrubicina, que é geralmente o tratamento usado para os casos de linfoma canino (Lee, Liao, \& Wang, 2020). A duração total do tratamento consiste em 19 semanas de tratamento (Chun, 2019), sendo uma variação mais curta do período de tempo do protocolo CHOP que varia de 15 a 25 semanas. (Thamm, 2019). Dados de literaturas indicam que o emprego de tratamentos quimioterápicos baseados no protocolo CHOP, apresentam uma taxa de remissão de 80-90\% com um tempo de sobrevida de 10 a 12 meses, o que faz esse protocolo ser substancialmente escolhido para o tratamento de linfoma multicêntrico (Burgess, 2020).

Outras opções de tratamento consistem na administração de ciclofosfamida, vincristina e prednisona denominado COP. Esse protocolo apesar de apresentar menor custo está associado a importantes efeitos colaterais como cistite hemorrágica estéril e extravasamento perivascular. Tais riscos são fatores que devem ser levados em consideração. Ademais, o COP apresenta um maior tempo de manutenção e estudos demonstram que o seu período de sobrevida é curto em relação a outros protocolos como o CHOP (Chun, 2019).

A utilização do protocolo com multiagentes é preferível em relação a administração única da prednisolona, sendo adotada neste caso clínico. O uso apenas desse corticosteróide melhora clinicamente e induz a remissão parcial de muitos cães com linfoma. Porém sua duração é de curto prazo (1-2 meses) e há a possibilidade de induzir resistência à quimioterapia, podendo reduzir seu sucesso terapêutico (Thamm, 2019). Outros protocolos que envolvem COP (sem doxorrubicina) ou LCHOP (com L-asparaginase) possibilitam maior sobrevida aos pacientes caninos do que apenas a utilização monodroga de prednisolona, por exemplo (Moore \& Frimberger, 2018).

É conhecido também que a adição da asparaginase ao protocolo COP não resulta em maior remissão clínica sobre o linfoma multicêntrico canino, porém há indicação de que esse fármaco seja mais adequado quando utilizado em tratamento de linfomas recidivantes (Jeffreys et al., 2005; Macdonald et al., 2005). Thamm et al. (2019) indicam utilizar a L-Asparaginase apenas na terapia de resgate e não na terapia inicial dos linfomas, pois esse agente traz melhoras questionáveis sobre essa neoplasia.

A quimioterapia oral que envolve prednisona e lomustina apesar da praticidade e fácil adesão pelos tutores apresenta baixo sucesso terapêutico nos casos de linfoma canino, exceto o cutâneo. Linfomas indolentes ou de baixo grau apresentam boa resposta a protocolos com prednisolona e clorambucil por via oral. Porém, nenhum protocolo quimioterápico oral é 
eficiente em linfomas multicêntricos de intermediário e alto graus (Thamm, 2019). Por tais considerações esse tipo de quimioterapia foi descartado.

Outros protocolos que apresentam respostas tão boas como o protocolo CHOP quanto à sobrevida e remissão dessa doença envolvem a combinação de lomustina, vincristina, procarbazina e prednisolona (protocolo LOPP). Esta combinação apresenta-se bastante efetiva em linfomas multicêntricos de alto grau e possui mínimos efeitos tóxicos (hepatotoxicidade, mielotoxicidade e gastrintestinais) (Brown, Tzannes, Nguyen, White, \& Langova, 2017). Tais características deste protocolo são extremamente úteis principalmente quando os pacientes acometidos apresentam comorbidades, o que não era o caso do animal acompanhado que recebeu o protocolo CHOP. No entanto, sabe-se que quando aumentadas as doses dos fármacos LOPP, apesar de aumentar a toxicidade, aumenta-se a sobrevida dos pacientes e o tempo livre de doença (Morgan, O_. Connell, Thomson, \& Griffin, 2018).

Assim como relatado por Hosoya et al. (2007) o protocolo CHOP apresenta melhor eficiência em relação a outros protocolos como COAP, porém apresenta maiores ocorrências de efeitos adversos como neutropenia e toxicidade gastrointestinal. De fato, no caso do animal abordado apresentou excelente resposta ao tratamento instituído, visto que não houve recidiva da neoplasia até 7 meses após o tratamento. Apesar dos ótimos resultados com o protocolo CHOP a sua toxicidade é levada em consideração devido à doxorrubicina. Porém, sabe-se que isso não é um fator que inviabilize o seu uso, inclusive a hematotoxicidade e cardiotoxicidade não tem diferenças significativas com outros protocolos como aqueles que substituem a doxorrubicina por mitoxantrona (Wang, Lee \& Liao, 2016). Assim, o emprego do protocolo Wisconsin-Madison é viável a este caso por ser indicado para o tratamento de linfoma em caninos, com resultados satisfatórios. A sua duração de tratamento rápida acaba sendo mais viável em logística ao tutor visto que fatores financeiros são peças limitantes para o emprego da quimioterapia de forma efetiva e completa.

\section{Conclusão}

O aprimoramento e adequação do protocolo quimioterápico utilizado para cada animal é de fundamental importância para um prognóstico bom e com baixos efeitos colaterais. A escolha do protocolo Madison-Wisconsin para o tratamento do linfoma multicêntrico em um cão foi satisfatório na remissão completa dessa neoplasia. Novos estudos podem ser realizados para uma maior compreensão da efetividade desse protocolo no tratamento desta patologia.

\section{Referências}

Archer, T., \& Sullivant, A. (2018). Top 5 Causes of Splenomegaly in Dogs. https://www.cliniciansbrief.com/article/top-5-causes-splenomegaly-dogs

Beraldo, M. R. A., Varzim, F. L. S. B., \& Pulz, L. H. (2020). Linfoma Multicêntrico Canino: uma sinopse sobre os aspectos clinicopatológicos e alterações laboratoriais. Revista de Educação Continuada em Medicina Veterinária e Zootecnia do CRMV-SP, 18(2).

Biller, B., Berg, J., Garrett, L., Ruslander, D., W earing, R., Abbott, B., P., \&. Bryan, C. (2016). AAHA Oncology Guidelines for Dogs and Cats. American Animal Hospital Association, 52(4), 181-204.

Brown, P. M., Tzannes, S., Nguyen, S., White, J., \& Langova, V. (2017). LOPP chemotherapy as a first-line treatment for dogs with T-cell lymphoma. Veterinary and Comparative Oncology, 16(1), 108-113.

Bruyette, D. S. (2020). Lymphomas. In K.E. Burgess (Org.), Clinical Small Animal Internal Medicine (Cap. 134, pp. 1231-1239). USA: John Wiley \& Sons, Inc.

Burton, J. H., Garret-Mayer, E., \& Thamm, D. H. (2012). Evaluation of a 15-week CHOP protocol for the treatment of canine multicentric lymphoma. Veterinary and Comparative Oncology, 11(4), 306-315.

Cápua, M. L. B., Coleta, F. E. D., Canesin, A. P. M. N., Godoy, A.V., Calazans, S. G., Miotto, M. R., Daleck, C. R., \& Santana, A. E. (2011). Linfoma canino: clínica, hematologia e tratamento com o protocolo de Madison-Wisconsin. Ciência Rural, 41(7), 1245-1251.

Chun, R. (2009). Lymphoma: which chemotherapy protocol and why?. Topics in companion animal medicine, 24(3), 157-162.

Daleck, C. R., \& De Nardi, A. B. (2016). Oncologia em cães e gatos. (2a ed.). Roca. 
Eberhardt, F., Köhler, C., Krastel, D., Winter, K., Alef, M., \& Kiefer, I. (2015). Sonographisch erfassbare Milzveränderungen bei Hunden mit malignem Lymphom [Sonographically detectable splenic disorders in dogs with malignant lymphoma]. Tierarztliche Praxis, 43(4), 215-220.

Flory, A. B., Rassnick, K. M., Erb, H. N., Garrett, L. D., Northrup, N. C., Selting, K. A., P..., \& Chretin, J. D. (2011). Evaluation of factors associated with second remission in dogs with lymphoma undergoing retreatment with a cyclophosphamide, doxorubicin, vincristine, and prednisone chemotherapy protocol: 95 cases (2000-2007). J Am Vet Med Assoc., 238(4), 501-506.

Garrett, L. D., Thamm, D. H.; Chun, R.; Dudley, R., \& Vail, D. M. (2002, Junho, 28). Evaluation of a 6-month chemotherapy protocol with no maintenance therapy for dogs with lymphoma. Journal of Veterinary Internal Medicine, 16(6), 704-709.

Hosoya, K., Kisseberth, W. C., Lord, L. K., Alvarez, F. J., Lara-Garcia, A., Kosarek, C. E., \& Couto, C. G. (2007). Comparison of COAP and UW-19 Protocols for Dogs with Multicentric Lymphoma. J Vet Intern Med. 21(6), 1355-1363.

Jeffreys, A. B., Knapp, D. W., Carlton, W. W., Thomas, R. M., Bonney, P. L., D...., \& Lucroy, M. D. (2005). Influence of asparaginase on a combination chemotherapy protocol for canine multicentric lymphoma. Journal of the American Animal Hospital Association, 41(4), 221-226.

Jia, S.S., Hou, Z.Z, H., Meng, B., \& Ma, B.D. (2016). Diagnosis and pathological characteristics of canine lymphoma: report of 4 cases. Int J Clin Exp Pathol, 9(2), 2389-2395.

Laoharatchatathanin, T., Klomsatid, N., \& Thermtong, P. (2019). Multicentric Lymphoma in Fila Brasileiro Puppy: A Case Report. Journal of Mahanakorn Veterinary Medicine, 14(supplement), pp. 23-31.

Lee, J. J., Liao, A. T., \& Wang, S. L. (2020). Outcome of Canine Multicentric Lymphoma after Single or Divided Treatment with Cyclophosphamide in Multidrug Chemotherapy. Topics in companion animal medicine, $41,100461$.

Macdonald, V. S., Thamm, D. H., Kurzman, I. D., Turek, M. M., \& Vail, D. M. (2005). Does L-asparaginase influence efficacy or toxicity when added to a standard CHOP protocol for dogs with lymphoma? Journal of veterinary internal medicine, 19(5), 732-736.

Marconato, L. (2011). The staging and treatment of multicentric high-grade lymphoma in dogs: A review of recent developments and future prospects. The Veterinary Journal, 188(1), 34-38.

Mazaro, R. D., Rizkallah, I. P. J., Luz, F. S., Lorensetti, D. M., Cogliati, B., \& Fighera, R. A. (2018). Aspectos epidemiológicos, clínicos e anatomopatológicos do linfoma folicular em cães. Pesquisa Veterinária Brasileira., 38(9), 1772-1780.

Moore, A. S., \& Frimberger, A. E. (2018). Usefulness of chemotherapy for the treatment of very elderly dogs with multicentric lymphoma. Journal of the American Veterinary Medical Association, 252(7), 852-859.

Morgan, E., O'connell, K., Thomson, M., \& Griffin, A. (2018). Canine T cell lymphoma treated with lomustine, vincristine, procarbazine, and prednisolone chemotherapy in 35 dogs. Veterinary and comparative oncology, 16(4), 622-629.

Neel, J. A., Synder, L., \& Grindem, C. B. (2012). Thrombocytosis: a retrospective study of 165 dogs. Veterinary Clinical Pathology, 41(2), $216-222$.

Nelson, R. W. \& Couto, C. G. (2015). Medicina interna de pequenos animais (5a ed). Elsevier.

Oliveira, F. M. (2019). Linfoma multicêntrico em cão - relato de caso. (Trabalho de Conclusão de Curso). Universidade Federal de Santa Catarina, Curitibanos, Brasil.

Pereira, B. J., Nunes, L. C., Filho, S. M., \& Costa, F. S. (2011). Avaliação dos efeitos da terapia com prednisona em cães utilizando análises ultrassonográficas, citopatológica e histopatológica. Revista Ceres Viçosa, 58(5), 561-566.

Silveira, P. S. D. (2016). Efeitos secundários da quimioterapia antineoplásica e seu impacto na qualidade de vida em cães e gatos com doença oncológica. Dissertação (Mestrado). Faculdade de Medicina Veterinária, Universidade de Lisboa.

Thamm, D. H. (2019). Novel Treatments for Lymphoma. The Veterinary clinics of North America. Small animal practice, 49(5), $903-915$.

Vail, D. M., Thamm, D.H., \& Liptak, J. M. (2020). Withrow and MacEwen's Small Animal Clinical Oncology. (6a ed.). Mo. :Saunders Elsevier.

Wang, S. L., Lee, J. J., \& Liao, A. T. (2016). Comparison of efficacy and toxicity of doxorubicin and mitoxantrone in combination chemotherapy for canine lymphoma. The Canadian veterinary journal,, 57(3), 271-276.

Zamokas, G., Grigonis, A., Babickaitè, L., Riłkevičienè, V., Lasienė, K. \& Juodžiukynienè, N. (2016). Extramedullary hematopoiesis (EMH) and other pathological conditions in canine spleens. Med. Weter, 72(12), 768-772.

Zandvliet, M. (2016). Canine Lymphona: a review. Veterinary Quarterly. 36(2), 76-104. 